\section{Neural stem cells aplenty}

\section{By Kai-Jye Lou, Staff Writer}

Sloan-Kettering Institute researchers have developed a protocol for inducing neural cells that could alleviate some of the manufacturing and supply issues hindering the development of therapies, drug screening platforms and disease models that use human neural stem cells. ${ }^{1}$ In contrast to other methods, the protocol consists of a defined set of neural-inducing factors, which makes it more amenable to a GMP setting and decreases the time to convert embryonic and induced pluripotent stem cells into neural stem cells.

The drawbacks of current protocols include the use of feeder cells that secrete undefined neural-induction factors, unpredictable results from the formation of multicellular aggregates known as embryoid bodies, low yields and lengthy induction times., ${ }^{2,3}$

"The problem with the use of undefined factors in your differentiation protocols is that you can have batch-to-batch variations-your product is less reliable," said Lorenz Studer, an associate member in developmental biology at Sloan-Kettering. "Some of these earlier differentiation protocols require feeders, which bring in foreign cells. These cells are a potential source of contamination and immunogenicity."

Neural stem cell induction protocols that rely on embryoid body formation also have reliability issues, said Studer. "The formation of an embryoid body is unpredictable by nature, so sometimes it works well and sometimes not so well," he said.

Two factors that have been used separately to induce neural conversion of human embryonic stem cells are Noggin, a protein-based bone morphogenetic protein (BMP) inhibitor, and SB431542, a small molecule inhibitor of activin receptor-like kinase (ALK) receptor phosphorylation. ${ }^{4,5}$ Both compounds inhibit signaling of a transcription factor called mothers against decapentaplegic homolog (SMAD; MADH).

As reported in a paper published in Nature Biotechnology, a research group led by Studer combined the two SMAD inhibitors in a protocol that addresses each drawback of current induction methods.

The group converted human embryonic and induced pluripotent stem cells into a homogeneous population of neuroectodermal precursor cells that produced neural stem cells. The induction protocol yielded about $82 \%$ neuroectodermal precursor cells after 13 days. The previous best induction method using Noggin and mouse stromal feeder cells had about a $26 \%$ yield at the same time point.
The researchers then used established cell differentiation protocols to convert the neuroectodermal precursors into cells that expressed markers for motor neurons and dopaminergic neurons. The induction and subsequent differentiation of embryonic stem cells into these neuronal cells required about 19 days. Other methods would have required 30-50 days. ${ }^{4,6}$

"One of the barriers to industrial adoption of neural stem cell-based tools and therapies is the time it takes to convert stem cells into these neuroectodermal precursors," Studer told SciBX.

Sally Temple, scientific director of the New York Neural Stem Cell Institute and a professor at Albany Medical College, said the speed of the Noggin/SB431542 protocol would indeed drive uptake of the technique.

"The protocol is very rapid - no longer do we have to wait 30 days to make neural cells," she noted. "I see this protocol being adopted by any lab using embryonic or induced pluripotent stem cells to make neural cell types."

In addition to getting neural cells in short order, the induction protocol also should expedite the development of new differentiation protocols. "Previously, it would take a year or two to develop a protocol that generates a highly specific neural subtype from stem cells. With this protocol, we can do it in several months," Studer said. "The dual SMAD inhibition protocol therefore increases the applicability of stem cells to disease modeling and to largescale drug toxicology and screening studies."

\section{Quick and easy}

Studer thinks the protocol works by promoting the loss of pluripotency in human embryonic stem cells and preventing their differentiation into non-neuroectodermal cell types (see Figure 1, "Model for generating neural stem cells"). The key, he said, is using only two factors-Noggin and SB431542 - to get the desired results.

Because those two factors are known, "it will be easier to eventually adapt this protocol into a manufacturing process that meets GMP standards" compared with feeder cells, which secrete undefined neural-induction factors, Studer said.

Studer did note that the work on converting embryonic and induced pluripotent stem cells into neuronal cells is only halfway done. "The goal in this field is not just to make neural stem cells, but also to make all the types of neural cells relevant to each disease type," he said, such as dopaminergic neurons for Parkinson's disease (PD) and motor neurons for amyotrophic lateral sclerosis (ALS).

He added that his group and others are trying to develop the protocols and define the factors needed to differentiate the neuroectodermal precursors into various neuronal cell types.

Other researchers are eager to see such results. Arnold Kriegstein, director of the Eli and Edythe Broad Center of Regeneration Medicine and Stem Cell Research at the University of California, San Francisco, wanted to know if the neuroectodermal precursors generated with this 
Figure 1. Model for generating neural stem cells. In Nature Biotechnology, researchers showed that SB431542 and Noggin can be used to convert embryonic and induced pluripotent stem cells into neuroectodermal cells by blocking the signaling of a transcription factor called mothers against decapentaplegic homolog (SMAD; $\mathrm{MADH})$.

[a] SB431542 inhibits transforming growth factor- $\beta$ (TGF- $\beta$ ), activin receptor-like kinase (ALK) and NODAL signaling, which are necessary for maintaining pluripotency in human embryonic stem cells.

The inhibition of ALK and NODAL signaling prevents embryonic stem cells from differentiating into mesendodermal cells.

[b] Noggin inhibits bone morphogenetic protein (BMP), which prevents embryonic stem cells from differentiating into trophectodermal and ectodermal cells.

[c] By blocking differentiation into mesendodermal, trophectodermal and ectodermal cells, embryonic stem cells differentiate into paired box 6 (PAX6) $)^{+}$, and p75+ and $\beta-1,3-$ glucuronyltransferase 1 (B3GAT1; HNK-1) neuroectodermal precursor cells. The ratio between the two cell types depends on the initial density of embryonic stem cells, with higher densities biasing differentiation towards PAX6 ${ }^{+}$cells. p $75^{+}$and $\mathrm{HNK}-1^{+}$cells are usually found with PAX6 ${ }^{+}$cells.

[d; e; f] Researchers can use cell passage and/or patterning protocols to differentiate PAX6 $6^{+}$and $\mathrm{p} 75^{+} / \mathrm{HNK}-1^{+}$cells into a variety of neural cells. PAX6 ${ }^{+}$cells are precursors to CNS-related cells, including rosette neural stem cells, whereas p $75^{+}$and $\mathrm{HNK}-1^{+}$cells are precursors to peripheral nervous system (PNS) cells and neural crest stem cells. $\mathrm{p} 75^{+}$and $\mathrm{HNK}-1^{+}$cells are also precursors to non-neuronal cells such as melanocytes.

Solid lines represent cellular fates shown by the researchers in the Nature Biotechnology paper. Dotted lines indicate potential cellular fates based on current literature.

protocol could be differentiated into glial cell types or spatially restricted cell types like forebrain-specific cells.

Because cell differentiability is time dependent, Temple said researchers adopting this protocol will need to learn the timing on when specific progenitor cell types arise. This will be important for knowing when the cells can be differentiated into a particular nervous system cell.

To support its adoption into an industrial context, Kriegstein said it will be important to show that the protocol can be scaled up.

Studer said that because Noggin is an expensive factor, he would like to replace it with a small molecule BMP inhibitor, which would further lower costs.

Sloan-Kettering has filed a provisional patent application covering the dual SMAD inhibition neural induction protocol. The work is available for licensing.

Lou, K.-J. SciBX 2(12); doi:10.1038/scibx.2009.476

Published online March 26, 2009

\section{REFERENCES}

1. Chambers, S.M. et al. Nat. Biotechnol.; published online March 1, 2009; doi:10.1038/nbt.1529

Contact: Lorenz Studer, Memorial Sloan-Kettering Cancer Center, New York, N.Y.

e-mail: studerl@mskcc.org

Contact: Stuart M. Chambers, same affiliation as above e-mail: chambers@mskcc.org

2. Schwartz, P.H. et al. Methods 45,142-158 (2008)

3. Cai, C. \& Grabel, L. Dev. Dyn. 236, 3255-3266 (2007)

4. Lee, H. et al. Stem Cells 25, 1931-1939 (2007)

5. Smith, J.R. et al. Dev. Biol. 313, 107-117 (2008)

6. Perrier, A.L. et al. Proc. Natl. Acad. Sci. USA 101, 12543-12548 (2004) 